\title{
A robust optimal trajectory tracking control for systems with an input delay
}

\author{
Yusheng Zhou, Zaihua Wang \\ State Key Laboratory of Mechanics and Control of Mechanical Structures \\ Nanjing University of Aeronautics and Astronautics, 210016 Nanjing, China
}

\begin{abstract}
This paper proposes a robust controller for the optimal trajectory tracking problem of linear systems with an input delay and subjected to an external disturbance. We introduce a new integral transformation to convert the original error system with time delay into a delay-free one, and observe a key but simple relationship between the original state variables and the new state variables. We design the robust controller in two parts: one is a delayed optimal trajectory tracking controller used for implementing the control task, and the other is a disturbance observer used for compensating the external disturbance. Due to the delay effect, the current state in the controller is replaced with a predictor state. For demonstration, a robust controller to control the Back-and-Forth motion of an inverted pendulum on a cart is designed, where the pitch angle of the inverted pendulum is forced to be small by choosing a large weight of the pitch angle error in the quadratic performance criterion. Simulation results show that the proposed controller not only implements the control task well but also has a strong robustness against the disturbance.
\end{abstract}

Keywords: Input delay, Optimal trajectory tracking control, Inverted pendulum, Predictor-based feedback, Disturbance observer, Back-and-Forth motion

\section{Introduction}

Trajectory tracking control has a number of important applications in high-tech fields, such as aircraft and space vehicle [1, 2], mobile robot [3, 4], tank system [5] and underwater vehicle [6, 7]. For a given reference trajectory, the control design requires to find an appropriate controller to track the reference trajectory with tracking error converging to zero. Thus, the trajectory tracking problem can be transformed into a stability problem of an error system. In many applications, trajectory tracking problems are subjected to additional requirements, such as to track the target within a shorter time or to consume less energy and small error sum. Thus, the well-developed optimal control theory $[8,9]$ is a natural choice in solving such tracking problems, such as in industrial production [10], ocean transportation [11, 12], radar tracking [13], missile tracking [14], aircraft and space vehicle [15, 16], robot control [17, 18] and so on. In the literature, optimal control theory is hardly used alone but usually applied for the trajectory tracking control problems as shown in the above mentioned examples.

With the use of a large number of digital controllers and filters in trajectory tracking control problems, the inevitable delay effect must be taken into account. An input delay is the time gap in the control path from the time when measuring signal of the control plant begins, to the time when the control takes effect to the closed-loop. The main negative impact of the input delay is that the feedback state is delayed, and the available feedback information to determine the controller is the delayed feedback state. As shown in $[19,20]$, an input delay caused by controllers and

\footnotetext{
Corresponding author: Zaihua Wang.

Email addresses: sands1119@126. com (Yusheng Zhou), zhwang@nuaa.edu.cn (Zaihua Wang)
} 
filters, though very small, leads to destabilization or bad performance. In addition, the delay results in complicated analysis and design because a delayed system is indefinite dimensional, no matter how small the delay is. Thus, the delay is usually ignored in many engineering applications due to its small quantity. Theoretically, LyapunovKrasovskii functional method and linear matrix inequality (LMI) technique are used frequently in the literature [21, 22], but they usually lead to conservative results. In many applications, an integral transformation of the states [23, 24] is usually adopted to transform the input delay system into a delay-free one, so that the control design can be made by using the delay-free system rather than the original system. This reduction method works for linear controlled systems, it can not used to deal with nonlinear systems with an input delay.

In addition, uncertainty coming from modelling, measurement and external disturbance is also inevitable in practical applications and must be taken into account in the design phase. Kalman filter method and its generalization such as unscented Kalman filter method have been widely used to estimate the internal and external uncertain parameters, but they work for linear systems or weak nonlinear systems only [25]. Moreover, optimal control is based on the precise system model, and it has weak robustness against uncertainty. Thus, little attention has been paid to the optimal trajectory tracking control problems, when uncertainty has to be considered. This motivates us to develop a design method for the trajectory tracking control problems, subjected to both uncertainty and optimal conditions.

Nonlinearity is another important factor that has to be considered in many applications. Though nonlinearity exists commonly in practical systems and great progress has been achieved in nonlinear control theory, the control design of nonlinear systems is usually based on some properly linearized systems. Feedback linearization is one popular method for nonlinear control problems [26], but it requires complicated computation when the order of the system is high. Intuitively, Optimal control strategy can be powerful in some nonlinear control problems. Actually, under the frame of LQR control, the performance criterion to be minimized has clear physical meaning, it contains a term of energy consumption and a term of state error of the system model. With a weight matrix whose elements are large enough in the performance criterion, the corresponding state error can be small enough, and the optimal control forces the tracking state approaching to the trajectory tracking target moment by moment. In this way, the control design can be based on the linearized system, regarding the modelling error as external disturbance. This is particular useful when the controlled system has strong nonlinearity. Though the robustness of the optimal controller can be destroyed by uncertainty, it can be improved by adding a disturbance observer. Therefore, the designed controller is suboptimal.

In this paper, we propose a design method for the robust optimal trajectory tracking control of linear delayed-input systems with an external disturbance. Problem statement and some preliminaries are first given in Section 2. Then a delayed optimal controller is designed for the tracking error systems with a known disturbance in Section 3, and a disturbance observer which is introduced to compensate the unknown disturbance is given in Section 4. Next, some implementation problems of the controller are discussed in Section 5. As an application, the delayed optimal trajectory tracking controller of the Back-and-Forth motion of an inverted pendulum on a cart (a nonlinear system) is given in Section 6. Finally some concluding remarks are made in Section 7.

\section{Problem statement and preliminaries}

\subsection{Problem statement}

Let us consider a linear system with an input delay and a known disturbance

$$
\dot{x}(t)=A x(t)+B u(t-\tau)+v(t)
$$




\begin{tabular}{|l|l|l|l|}
\hline Table 1: Main Notations \\
\begin{tabular}{|l|l|l|l|}
\hline Notation & Definition & Notation & Definition \\
\hline$x(t)$ & Original state variable & $A, B$ & Matrices \\
\hline $\bar{x}(t)$ & Reference trajectory tracking target & $\tau$ & Input delay \\
\hline$y(t)$ & Error variable $y(t):=x(t)-\bar{x}(t)$ & $v(t)$ & External disturbance \\
\hline$z(t)$ & New state variable of the delay-free system & $u(t)$ & Control variable \\
\hline$Q_{0}, Q, R$ & Weight matrices in quadratic performance criterion & $\omega(t)$ & $\omega(t):=A \bar{x}(t)-\dot{\bar{x}}(t)$ \\
\hline$H$ & Hamiltonian function & $\tilde{v}(t)$ & Estimated $v(t)$ \\
\hline$H_{0}$ & Hamiltonian matrix & $B^{T}$ & Transpose of $B$ \\
\hline
\end{tabular}
\end{tabular}

with the initial condition $x(0)=x_{0}$. Here, $x(t) \in \mathbb{R}^{n}$ is the system state and is completely measurable, $u(t) \in \mathbb{R}^{m}$ is the control variable, $\tau$ is the input delay and is a known constant, $v(t) \in \mathbb{R}^{n}$ is the known external disturbance vector, the pair of constant matrices $(A, B)$ is controllable, and the control takes place when $t \geq \tau$.

Assume $\bar{x}(t)$ is an arbitrary tractable reference trajectory tracking target, and $y(t):=x(t)-\bar{x}(t)$ is the tracking error. Let $\omega(t):=A \bar{x}(t)-\dot{\bar{x}}(t)$, then system (1) can be transformed into the following form

$$
\left\{\begin{array}{l}
\dot{y}(t)=A y(t)+B u(t-\tau)+v(t)+\omega(t) \\
y(0)=x(0)-\bar{x}(0)
\end{array}\right.
$$

The objective is to find a delayed optimal tracking control $u^{*}(t-\tau)$ that minimizes the quadratic performance criterion

$$
J=\frac{1}{2} y^{\mathrm{T}}\left(t_{f}\right) Q_{0} y\left(t_{f}\right)+\frac{1}{2} \int_{0}^{t_{f}}\left[y^{\mathrm{T}}(t) Q y(t)+u^{\mathrm{T}}(t-\tau) R u(t-\tau)\right] \mathrm{d} t
$$

where $Q_{0}, Q$ are nonnegative definite matrices, $R$ is a positive definite matrix, and $t_{f}(>2 \tau)$ is the terminal time.

\subsection{Preliminaries about optimal controller for systems without input delay}

To solve this problem, let us firstly recall some facts for the linear system (2) with $\tau=0$. Let the augmented quadratic performance criterion be defined as

$$
\bar{J}=\frac{1}{2} y^{\mathrm{T}}\left(t_{f}\right) Q_{0} y\left(t_{f}\right)+\frac{1}{2} \int_{0}^{t_{f}}\left[y^{\mathrm{T}}(t) Q y(t)+u^{\mathrm{T}}(t) R u(t)\right] \mathrm{d} t+\int_{0}^{t_{f}}\left[\lambda^{\mathrm{T}}(t)(A y(t)+B u(t)+v(t)+\omega(t)-\dot{y}(t))\right] \mathrm{d} t
$$

and the Hamiltonian function is defined by

$$
H(y, u, \lambda, t)=\frac{1}{2} y^{\mathrm{T}}(t) Q y(t)+\frac{1}{2} u^{\mathrm{T}}(t) R u(t)+\lambda^{\mathrm{T}}(t)(A y(t)+B u(t)+v(t)+\omega(t))
$$

Then $\bar{J}$ can be rewritten in the form of

$$
\begin{aligned}
\bar{J} & =\frac{1}{2} y^{\mathrm{T}}\left(t_{f}\right) Q_{0} y\left(t_{f}\right)+\int_{0}^{t_{f}}\left[H(y, u, \lambda, t)-\lambda^{\mathrm{T}}(t) \dot{y}(t)\right] \mathrm{d} t \\
& =\frac{1}{2} y^{\mathrm{T}}\left(t_{f}\right) Q_{0} y\left(t_{f}\right)-\lambda^{\mathrm{T}}\left(t_{f}\right) y\left(t_{f}\right)+\lambda^{\mathrm{T}}(0) y(0)+\int_{0}^{t_{f}}\left[H(y, u, \lambda, t)+\dot{\lambda}^{\mathrm{T}}(t) y(t)\right] \mathrm{d} t
\end{aligned}
$$

The variation of $\bar{J}$ with respect to $u$ and $y$ is found to be

$$
\delta \bar{J}=\delta y^{\mathrm{T}}\left(t_{f}\right) Q_{0} y\left(t_{f}\right)-\delta y^{\mathrm{T}}\left(t_{f}\right) \lambda\left(t_{f}\right)+\int_{0}^{t_{f}}\left[\delta y^{\mathrm{T}}\left(\frac{\partial H}{\partial y}+\dot{\lambda}(t)\right)+\delta u^{\mathrm{T}} \frac{\partial H}{\partial u}\right] \mathrm{d} t
$$


By setting $\delta \bar{J}=0$, the necessary conditions for the minimum value problem are

$$
\left\{\begin{array}{l}
\dot{\lambda}(t)=-\frac{\partial H}{\partial y} \\
\dot{y}(t)=\frac{\partial H}{\partial \lambda} \\
\frac{\partial H}{\partial u}=0 \\
\lambda\left(t_{f}\right)=Q_{0} y\left(t_{f}\right)
\end{array}\right.
$$

From Eq.(4) and Eq.(5), the optimal control of system (2) that minimizes performance criterion (3) is given by

$$
u^{*}(t)=-R^{-1} B^{\mathrm{T}} \lambda(t)
$$

where $\lambda(t)$ is determined by

$$
\left\{\begin{array}{l}
-\dot{\lambda}(t)=Q y(t)+A^{\mathrm{T}} \lambda(t), \quad \lambda\left(t_{f}\right)=Q_{0} y\left(t_{f}\right) \\
\dot{y}(t)=A y(t)-B R^{-1} B^{\mathrm{T}} \lambda(t)+v(t)+\omega(t), \quad y(0)=x(0)-\bar{x}(0)
\end{array}\right.
$$

Lemma 1. When $\tau=0$, the optimal control of system (2) that minimizes $J$ in (3) is given by

$$
u^{*}(t)=-R^{-1} B^{\mathrm{T}}\left[P_{0}(t) y(t)+b_{0}(t)\right]
$$

where $P_{0}(t) \in \mathbb{R}^{n \times n}$ and $b_{0}(t) \in \mathbb{R}^{n}$ are the solutions of the Riccati differential equations

$$
\left\{\begin{array}{l}
\dot{P}_{0}(t)=-P_{0}(t) A-A^{\mathrm{T}} P_{0}(t)+P_{0}(t) B R^{-1} B^{\mathrm{T}} P_{0}(t)-Q, \quad P_{0}\left(t_{f}\right)=Q_{0} \\
\dot{b}_{0}(t)=-\left[A-B R^{-1} B^{\mathrm{T}} P_{0}(t)\right]^{\mathrm{T}} b_{0}(t)-P_{0}(t)(v(t)+\omega(t)), \quad b_{0}\left(t_{f}\right)=0
\end{array}\right.
$$

respectively, and the optimal value of $J$ reads

$$
J=J_{d}=\frac{1}{2} y^{\mathrm{T}}(0) P_{0}(0) y(0)+b_{0}^{\mathrm{T}}(0) y(0)+\frac{1}{2} c_{0}(0)
$$

where $c_{0}(t)$ is the solution of the following equation

$$
\dot{c}_{0}(t)=b_{0}^{\mathrm{T}}(t) B R^{-1} B^{\mathrm{T}} b_{0}(t)-2 b_{0}^{\mathrm{T}}(t)[v(t)+\omega(t)], \quad c_{0}\left(t_{f}\right)=0
$$

Proof: Firstly, let

$$
\lambda(t)=P_{0}(t) y(t)+b_{0}(t)
$$

Differentiating at the both sides of Eq.(9) and combining Eq.(6) lead to

$$
\begin{aligned}
\dot{\lambda}(t) & =\dot{P}_{0}(t) y(t)+P_{0}(t) \dot{y}(t)+\dot{b}_{0}(t) \\
& =\dot{P}_{0}(t) y(t)+P_{0}(t)\left(A y(t)-B R^{-1} B^{\mathrm{T}} \lambda(t)+v(t)+\omega(t)\right)+\dot{b}_{0}(t) \\
& =-Q y(t)-A^{\mathrm{T}} \lambda(t)
\end{aligned}
$$

Equating the corresponding coefficients of both sides of Eq.(10), one has the Riccati equation (8).

Next, let $F_{0}(t)=y^{\mathrm{T}}(t) P_{0}(t) y(t)+2 b_{0}^{\mathrm{T}}(t) y(t)+c_{0}(t)$, then we have

$$
\frac{\mathrm{d}}{\mathrm{d} t}\left[F_{0}(t)\right]=\dot{y}^{\mathrm{T}}(t) P_{0}(t) y(t)+y^{\mathrm{T}}(t) \dot{P}_{0}(t) y(t)+y^{\mathrm{T}}(t) P_{0}(t) \dot{y}(t)+2 \dot{b}_{0}^{\mathrm{T}}(t) y(t)+2 b_{0}^{\mathrm{T}}(t) \dot{y}(t)+\dot{c}_{0}(t)
$$


With $u(t)=u^{*}(t)=-R^{-1} B^{\mathrm{T}} \lambda(t)=-R^{-1} B^{\mathrm{T}}\left[P_{0}(t) y(t)+b_{0}(t)\right]$, Eq.(11) is changed to

$$
\frac{\mathrm{d}}{\mathrm{d} t}\left[y^{\mathrm{T}}(t) P_{0}(t) y(t)+2 b_{0}^{\mathrm{T}}(t) y(t)+c_{0}(t)\right]=-y^{\mathrm{T}}(t) Q y(t)-u^{\mathrm{T}}(t) R u(t)
$$

By integrating both sides of Eq.(12) from 0 to $t_{f}$ and multiplying both sides by $\frac{1}{2}$, we get

$$
\left.\frac{1}{2}\left[y^{\mathrm{T}}(t) P_{0}(t) y(t)+2 b_{0}^{\mathrm{T}}(t) y(t)+c_{0}(t)\right]\right|_{0} ^{t_{f}}=-\frac{1}{2} \int_{0}^{t_{f}}\left[y^{\mathrm{T}}(t) Q y(t)+u^{\mathrm{T}}(t) R u(t)\right] d t
$$

It follows that with $\tau=0$, we have

$$
\begin{aligned}
J_{d} & =\frac{1}{2} y^{\mathrm{T}}\left(t_{f}\right) Q_{0} y\left(t_{f}\right)-\left.\frac{1}{2}\left[y^{\mathrm{T}}(t) P_{0}(t) y(t)+2 b_{0}^{\mathrm{T}}(t) y(t)+c_{0}(t)\right]\right|_{0} ^{t_{f}} \\
& =\frac{1}{2} y^{\mathrm{T}}(0) P_{0}(0) y(0)+b_{0}^{\mathrm{T}}(0) y(0)+\frac{1}{2} c_{0}(0)
\end{aligned}
$$

It implies that the control $u^{*}(t)$ given by Eq.(7) minimizes $J$ given in (3). This complete the proof of Lemma 1.

From Lemma 1, we see that the optimal control $u^{*}(t)$ consists of two parts, $-R^{-1} B^{\mathrm{T}} P_{0}(t) y(t)$ is a feedback control that is determined by the current state, and the rest $-R^{-1} B^{\mathrm{T}} b_{0}(t)$ is a feedforward that is determined by the disturbance and tracking target.

\section{Optimal trajectory tracking control for delayed-input systems with a known disturbance}

\subsection{A new integral transformation and a key relationship between the state variables}

Now, we consider the case of $\tau \neq 0$. In order to convert system (2) into a delay-free one, let us introduce a new integral state transformation as follows

$$
z(t)=y(t)+\int_{t-\tau}^{t} \mathrm{e}^{-A(s-t+\tau)}[B u(s)+v(s+\tau)+\omega(s+\tau)] \mathrm{d} s
$$

With this transformation, system (2) is changed into a delay-free form

$$
\dot{z}(t)=A z(t)+B_{0} u(t)+G(t)
$$

where $B_{0}=\mathrm{e}^{-A \tau} B, G(t)=\mathrm{e}^{-A \tau}[v(t+\tau)+\omega(t+\tau)]$.

System (2) has an equivalent form as follows

$$
y(t)=\mathrm{e}^{A t} y(0)+\int_{0}^{t} \mathrm{e}^{A(t-s)}[B u(s-\tau)+v(s)+\omega(s)] \mathrm{d} s
$$

and the solution $y(t)$ satisfies

$$
\begin{aligned}
& y(t+\tau)=\mathrm{e}^{A \tau}\left(y(t)+\int_{t}^{t+\tau} \mathrm{e}^{A(t-s)}[B u(s-\tau)+v(s)+\omega(s)] \mathrm{d} s\right) \\
& =\mathrm{e}^{A \tau}\left(y(t)+\int_{t-\tau}^{t} \mathrm{e}^{-A(s-t+\tau)}[B u(s)+v(s+\tau)+\omega(s+\tau)] \mathrm{d} s\right) \\
& =\mathrm{e}^{A \tau} z(t)
\end{aligned}
$$

Thus, the initial condition $y(0)=y_{0}$ for system (2) is changed into $z(0)=\mathrm{e}^{-A \tau} y(\tau)$.

Eq.(15) plays a key role in the following analysis. It implies that the transformation (13) does not change the stability of system (2). Hence, we have 
Lemma 2. The asymptotic stability of $y(t)$ implies the asymptotic stability of $z(t)$, and vise versa.

Moreover, the quadratic performance criterion $J$ for system (2) can be decomposed by

$$
J=J_{1}+J_{2}
$$

where $J_{1}=\frac{1}{2} \int_{0}^{\tau} y^{\mathrm{T}}(t) Q y(t) \mathrm{d} t$ is fixed because the control does not take effect when $t \in[0, \tau)$, and

$$
J_{2}=\frac{1}{2} y^{\mathrm{T}}\left(t_{f}\right) Q_{0} y\left(t_{f}\right)+\frac{1}{2} \int_{0}^{t_{f}-\tau}\left[y^{\mathrm{T}}(t+\tau) Q y(t+\tau)+u^{\mathrm{T}}(t) R u(t)\right] \mathrm{d} t
$$

Hence, $J=J_{1}+J_{2}=\min$ if and only if $J_{2}=\min$. By substituting Eq.(15) into Eq.(16), the criterion $J_{2}$ takes the form

$$
J_{2}=\frac{1}{2} z^{\mathrm{T}}\left(t_{f}-\tau\right) \tilde{Q}_{0} z\left(t_{f}-\tau\right)+\frac{1}{2} \int_{0}^{t_{f}-\tau}\left[z^{\mathrm{T}}(t) \tilde{Q} z(t)+u^{\mathrm{T}}(t) R u(t)\right] \mathrm{d} t
$$

where $\tilde{Q}=\left(\mathrm{e}^{A \tau}\right)^{\mathrm{T}} Q \mathrm{e}^{A \tau}, \tilde{Q}_{0}=\left(\mathrm{e}^{A \tau}\right)^{\mathrm{T}} Q_{0} \mathrm{e}^{A \tau}$. Thus the original optimal control problem can be converted into the optimal control problem for linear delay-free system (14) associated with the performance criterion $J_{2}$.

\subsection{An optimal controller for systems (2) with an input delay}

According to Lemma 1, the optimal control of system (14) that minimizes the quadratic criterion $J_{2}$ is given by

$$
u^{*}(t)=-R^{-1} B_{0}^{\mathrm{T}}\left[P_{d}(t) z(t)+b_{d}(t)\right]
$$

where $P_{d}(t) \in \mathbb{R}^{n \times n}$ and $b_{d}(t) \in \mathbb{R}^{n}$ are the solutions of the Riccati differential equations

$$
\left\{\begin{array}{l}
\dot{P}_{d}(t)=-P_{d}(t) A-A^{\mathrm{T}} P_{d}(t)+P_{d}(t) B_{0} R^{-1} B_{0}^{\mathrm{T}} P_{d}(t)-\tilde{Q}, \quad P_{d}\left(t_{f}-\tau\right)=\tilde{Q}_{0} \\
\dot{b}_{d}(t)=-\left[A-B_{0} R^{-1} B_{0}^{\mathrm{T}} P_{d}(t)\right]^{\mathrm{T}} b_{d}(t)-P_{d}(t) G(t), \quad b_{d}\left(t_{f}-\tau\right)=0
\end{array}\right.
$$

By substituting Eq.(15) into Eq.(17), the delayed optimal control of system (2) can be expressed in terms of the state $y(t)$, rather than $z(t)$, in the form of

$$
u^{*}(t-\tau)=-R^{-1}\left(\mathrm{e}^{-A \tau} B\right)^{\mathrm{T}}\left[P_{d}(t-\tau) \mathrm{e}^{-A \tau} y(t)+b_{d}(t-\tau)\right], \quad t \in\left[\tau, t_{f}\right]
$$

Lemma 3. Let $u^{*}(t-\tau)$ be the delayed optimal control of system (2) that minimizes the performance criterion in (3), then

$$
u^{*}(t-\tau)=-R^{-1} B^{\mathrm{T}}\left[P_{0}(t) y(t)+b_{0}(t)\right], \quad t \in\left[\tau, t_{f}\right]
$$

where $P_{0}(t) \in \mathbb{R}^{n \times n}$ and $b_{0}(t) \in \mathbb{R}^{n}$ are the solutions of the Riccati differential equation (8).

Proof: By multiplying the first equation of Eq.(8) by $\left(\mathrm{e}^{A \tau}\right)^{\mathrm{T}}$ from the left and by $\mathrm{e}^{A \tau}$ from the right respectively, one see that $V_{1}(t)=\left(\mathrm{e}^{A \tau}\right)^{\mathrm{T}} P_{0}(t) \mathrm{e}^{A \tau}$ is the solution of the following differential equation

$$
\dot{V}_{1}(t)=-V_{1}(t) A-A^{\mathrm{T}} V_{1}(t)+V_{1}(t) B_{0} R^{-1} B_{0}^{\mathrm{T}} V_{1}(t)-\tilde{Q}
$$

subject to $V_{1}\left(t_{f}\right)=\tilde{Q}_{0}$. Replacing $t$ with $t-\tau$ in the first equation of equation (18) leads to

$$
\dot{P}_{d}(t-\tau)=-P_{d}(t-\tau) A-A^{\mathrm{T}} P_{d}(t-\tau)+P_{d}(t-\tau) B_{0} R^{-1} B_{0}^{\mathrm{T}} P_{d}(t-\tau)-\tilde{Q}
$$

subject to $P_{d}\left(t_{f}-\tau\right)=\tilde{Q}_{0}$. It follows that both $V_{1}(t)$ and $P_{d}(t-\tau)$ satisfy the same differential equation under the same terminal condition. Thus, $V_{1}(t)$ and $P_{d}(t-\tau)$ must be the same, namely

$$
P_{d}(t-\tau)=\left(\mathrm{e}^{A \tau}\right)^{\mathrm{T}} P_{0}(t) \mathrm{e}^{A \tau}
$$


Similarly, multiplying the second equation of Eq. $(8)$ by $\left(\mathrm{e}^{A \tau}\right)^{\mathrm{T}}$ from the left, one see that $V_{2}(t)=\left(\mathrm{e}^{A \tau}\right)^{\mathrm{T}} b_{0}(t)$ is the solution of the following differential equation

$$
\dot{V}_{2}(t)=-\left[A-\mathrm{e}^{-A \tau} B R^{-1} B^{\mathrm{T}} P_{0}(t) \mathrm{e}^{A \tau}\right]^{\mathrm{T}} V_{2}(t)-\left(\mathrm{e}^{A \tau}\right)^{\mathrm{T}} P_{0}(t)(v(t)+\omega(t)),
$$

subject to $V_{2}\left(t_{f}\right)=0$. By substituting Eq.(21) into the second equation of Eq.(18) and replacing $t$ with $t-\tau$ lead to

$$
\dot{b}_{d}(t-\tau)=-\left[A-\mathrm{e}^{-A \tau} B R^{-1} B^{\mathrm{T}} P_{0}(t) \mathrm{e}^{A \tau}\right]^{\mathrm{T}} b_{d}(t-\tau)-\left(\mathrm{e}^{A \tau}\right)^{\mathrm{T}} P_{0}(t)(v(t)+\omega(t)),
$$

subject to $b_{d}\left(t_{f}-\tau\right)=0$. Hence, $V_{2}(t)$ and $b_{d}(t-\tau)$ must be the same, namely

$$
b_{d}(t-\tau)=\left(\mathrm{e}^{A \tau}\right)^{\mathrm{T}} b_{0}(t)
$$

By substituting Eq.(21) and Eq.(22) into Eq.(19), we have

$$
u^{*}(t-\tau)=-R^{-1} B^{\mathrm{T}}\left[P_{0}(t) y(t)+b_{0}(t)\right]
$$

This completes the proof.

Lemma 3 shows that the delayed optimal control is the same in form as the delay free case. However, the quantities of $y(t)$ are not the same for the two cases. For the delayed case, $y(t)$ depends on the delay value. Consequently, the controller and the cost function depend on the delay value, too.

Theorem 1. Assume that the linear system (2) is completely measurable and controllable, then the delayed optimal trajectory tracking controller that minimizes $J$ in (3) is given by

$$
u^{*}(t-\tau)=-R^{-1} B^{\mathrm{T}} b_{0}(t)-R^{-1} B^{\mathrm{T}} P_{0}(t) \Phi(t, \tau)\left[y(\tau)+\int_{\tau}^{t} \Phi(\tau, s)\left(v(s)+\omega(s)-B R^{-1} B^{\mathrm{T}} b_{0}(s)\right) \mathrm{d} s\right], t \in\left[\tau, t_{f}\right]
$$

and the optimal quadratic performance criterion can be written as

$$
J=J_{d}=J_{1}+\frac{1}{2} y^{\mathrm{T}}(\tau) P_{0}(\tau) y(\tau)+b_{0}^{\mathrm{T}}(\tau) y(\tau)+\frac{1}{2} c_{0}(\tau)
$$

where $P_{0}(t) \in \mathbb{R}^{n \times n}$ and $b_{0}(t) \in \mathbb{R}^{n}$ are the solutions of the Riccati differential equation $(8), K_{0}(t)=R^{-1} B^{\mathrm{T}} P_{0}(t)$ and $\Phi(t, \tau)$ is the state transition matrix of the following linear system

$$
\begin{aligned}
& \dot{y}(t)=\left(A-B K_{0}(t)\right) y(t), t \in\left[\tau, t_{f}\right) \\
& y(\tau)=\mathrm{e}^{A \tau}\left[y_{0}+\int_{0}^{\tau} \mathrm{e}^{-A s}(v(s)+\omega(s)) \mathrm{d} s\right]
\end{aligned}
$$

Proof: According to Lemma 3, the state $y(t)$ under the optimal control can be calculated step-by-step: when $t \in[0, \tau)$, the delayed optimal control does not work, thus the state of the system (2) without control reads

$$
y(t)=\mathrm{e}^{A t}\left[y_{0}+\int_{0}^{t} \mathrm{e}^{-A s}(v(s)+\omega(s)) \mathrm{d} s\right]
$$

Under the delayed optimal control function in the interval $\left[\tau, t_{f}\right]$, the state in the interval $\left[\tau, t_{f}\right]$ is found to be

$$
y(t)=\Phi(t, \tau)\left[y(\tau)+\int_{\tau}^{t} \Phi(\tau, s)\left(v(s)+\omega(s)-B R^{-1} B^{\mathrm{T}} b_{0}(s)\right) \mathrm{d} s\right]
$$

Substituting Eq.(25) into Eq.(20) results in the delayed optimal trajectory tracking controller (23). 
Applying Lemma 1 and Lemma 3, we find the optimal quadratic performance criterion $J_{d}$ as follows

$$
J_{d}=J_{1}+\frac{1}{2} z^{\mathrm{T}}(0) P_{d}(0) z(0)+b_{d}^{\mathrm{T}}(0) z(0)+\frac{1}{2} c_{d}(0)
$$

where $c_{d}(t)$ satisfies the following equation

$$
\dot{c}_{d}(t)=b_{d}^{\mathrm{T}}(t) B_{0} R^{-1} B_{0}^{\mathrm{T}} b_{d}(t)-2 b_{d}^{\mathrm{T}}(t) G(t), \quad c_{d}\left(t_{f}-\tau\right)=0
$$

From Eq.(21), Eq.(22) and Eq.(27), we have

$$
c_{d}(t-\tau)=c_{0}(t)
$$

Substituting Eq.(21), Eq.(22) and Eq.(28) into Eq.(26), then, the optimal performance criterion has its minimal value given in Eq.(24). This completes the proof.

Note that the optimal trajectory tracking control given by Eq.(23) is an open-loop control, and this might lead to bad robustness. In order to resolve this problem, a state feedback control is better than the open-loop control. However, due to the input delay, the feedback state is delayed, the only information of the feedback can be received is the delayed state $y(t-\tau)$. Hence, it is necessary to use a predictor state to replace the current state, so the original optimal trajectory tracking problem can be solved in the conventional way. Note that

$$
\begin{aligned}
y(t) & =\mathrm{e}^{A t} y(0)+\int_{0}^{t} \mathrm{e}^{A(t-s)}[B u(s-\tau)+v(s)+\omega(s)] \mathrm{d} s \\
& =\mathrm{e}^{A \tau} y(t-\tau)+\int_{t-\tau}^{t} \mathrm{e}^{A(t-s)}[B u(s-\tau)+v(s)+\omega(s)] \mathrm{d} s
\end{aligned}
$$

For the system model is completely precise, the current state $y(t)$ can be regarded as a predictor state $\bar{y}(t)$. Thus, Theorem 1 can be represented in the following form.

Theorem 2. Assume that the linear system (2) is completely measurable and controllable, then the delayed optimal trajectory tracking controller minimizes the quadratic performance criterion (3) is given by

$$
u^{*}(t-\tau)=-R^{-1} B^{\mathrm{T}}\left[P_{0}(t) \bar{y}(t)+b_{0}(t)\right], \quad t \in\left[\tau, t_{f}\right]
$$

where $\bar{y}(t)$ is the predictor state of $y(t)$

$$
\bar{y}(t)=\mathrm{e}^{A \tau} y(t-\tau)+\int_{t-\tau}^{t} \mathrm{e}^{A(t-s)}\left[B u^{*}(s-\tau)+v(s)+\omega(s)\right] \mathrm{d} s
$$

$P_{0}(t) \in \mathbb{R}^{n \times n}$ and $b_{0}(t) \in \mathbb{R}^{n}$ are the solutions of the Riccati differential equation (8).

The function $\omega(t)$ is determined by the known target. When the external disturbance $v(t)$ is precisely known, all the terms in the predictor $\bar{y}(t)$ are known. Moreover, solving the second equation in (8) gives:

$$
b_{0}(t)=-\Phi_{0}\left(t, t_{f}\right) \int_{t}^{t_{f}} \Phi_{0}\left(t_{f}, s\right) P_{0}(s) \omega(s) \mathrm{d} s-\Phi_{0}\left(t, t_{f}\right) \int_{t}^{t_{f}} \Phi_{0}\left(t_{f}, s\right) P_{0}(s) v(s) \mathrm{d} s
$$

where $\Omega(t):=-\left[A-B R^{-1} B^{\mathrm{T}} P_{0}(t)\right]^{\mathrm{T}}, \Phi_{0}\left(t, t_{f}\right)$ is the state transition matrix of system $\dot{w}(t)=\Omega(t) w(t)$. The key role of $b_{0}(t)$ in the optimal control (7) is to compensate the impacts from $\omega(t)$ and $v(t)$. By substituting the expression of $b_{0}(t)$ into Eq.(7), we can see that the optimal control quantity $u^{*}(t)$ is reduced when $v(t)$ has the same direction of $y(t)$, in this case, the external disturbance is beneficial. On the contrary, the optimal control quantity $u^{*}(t)$ is increased when $v(t)$ is in the opposite direction of $y(t)$. Therefore, if the external disturbance $v(t)$ is unknown, the optimal control does not exist. An additional element for compensating the disturbance has to be included to the controller such that the controller can be suboptimal. 


\section{Suboptimal trajectory tracking control for delayed-input systems with an unknown disturbance}

\subsection{Disturbance observer for compensating the unknown disturbance}

Disturbance observer is a natural choice to deal with the unknown external disturbance [27, 28]. In order to estimate the unknown disturbance $v(t)$ in the error system (2), let us introduce a disturbance observer as follows:

$$
\left\{\begin{array}{l}
\tilde{v}(t)=\xi(t)+C y(t) \\
\dot{\xi}(t)=-C \xi(t)-C(A y(t)+B u(t-\tau)+\omega(t)+C y(t)), \xi(0)=-C y(0)
\end{array}\right.
$$

where $\tilde{v}(t)$ is the estimated value of the unknown disturbance $v(t)$, and $C$ is a positive definite symmetric matrix.

Lemma 4. Assume that there exists a nonnegative constant $d$ such that $\|\dot{v}(t)\| \leq d$, then the disturbance observer (29) guarantees that the disturbance observer error $v_{e}(t)=v(t)-\tilde{v}(t)$ exponentially converges to a ball centered at the origin, and the radius of this ball can be made arbitrarily small by adjusting the matrix value $C$.

Proof: Let us first calculate the derivative of $\tilde{v}$ as follows

$$
\begin{aligned}
\dot{\tilde{v}}(t) & =-C \xi(t)-C(A y(t)+B u(t-\tau)+\omega(t)+C y(t))+C(A y(t)+B u(t-\tau)+v(t)+\omega(t)) \\
& =-C \xi(t)-C^{2} y(t)+C v(t) \\
& =-C \tilde{v}(t)+C v(t) \\
& =C v_{e}(t)
\end{aligned}
$$

Using (30), we have

$$
\dot{v}_{e}(t)=-C v_{e}(t)+\dot{v}(t)
$$

Consider the Lyapunov function

$$
V_{e}=\frac{1}{2} v_{e}^{\mathrm{T}}(t) v_{e}(t)
$$

whose first time derivative along the solution of (31) satisfies

$$
\begin{aligned}
\dot{V}_{e} & =-v_{e}^{\mathrm{T}}(t) C v_{e}(t)+v_{e}^{\mathrm{T}}(t) \dot{v}(t) \leq-\left(\lambda_{\min }(C)-\varepsilon\right) v_{e}^{\mathrm{T}}(t) v_{e}(t)+\frac{1}{4 \varepsilon} \dot{v}^{\mathrm{T}}(t) \dot{v}(t) \\
& \leq-2\left(\lambda_{\min }(C)-\varepsilon\right) V_{e}+\frac{1}{4 \varepsilon} d^{2}
\end{aligned}
$$

where $\lambda_{\min }(C)$ is the minimum eigenvalue of the matrix $C, \varepsilon$ is a positive constant such that $\lambda_{\min }(C)-\varepsilon$ is strictly positive. From (32), it is seen that $V_{e}$ exponentially converges to a ball centered the origin with the radius $R_{V}=$ $\frac{d^{2}}{8 \varepsilon\left(\lambda_{\min }(C)-\varepsilon\right)}$ as long as the solution $y(t)$ exist. This in turn means that the disturbance error $v_{e}$ exponentially converges to a ball centered at the origin with the radius $R_{v}=\sqrt{\frac{1}{4 \varepsilon\left(\lambda_{\min }(C)-\varepsilon\right)}} d$. Note that $\lambda_{\min }(C)$ can be chosen arbitrarily large by choosing the matrix $C$, the radius $R_{v}$ can be made arbitrarily small.

\subsection{Construction of the robust controller}

Although we cannot design an optimal control for the linear system (2) minimizes the performance criterion (3) when the external disturbance $v(t)$ is unknown, we can design a suboptimal control based on Theorem 2 and disturbance observer (29). Firstly, we consider the nominal system of the error system (2),

$$
\left\{\begin{array}{l}
\dot{y}(t)=A y(t)+B u(t-\tau)+\omega(t) \\
y(0)=x(0)-\bar{x}(0)
\end{array}\right.
$$


By using Theorem 2, the optimal control of such nominal system minimizes performance criterion (3) is given by

$$
u^{*}(t-\tau)=-R^{-1} B^{\mathrm{T}}\left[P_{0}(t) \bar{y}(t)+b_{0}(t)\right], \quad t \in\left[\tau, t_{f}\right]
$$

where $\bar{y}(t)$ is the predictor state of $y(t)$

$$
\bar{y}(t)=\mathrm{e}^{A \tau} y(t-\tau)+\int_{t-\tau}^{t} \mathrm{e}^{A(t-s)}\left[B u^{*}(s-\tau)+\omega(s)\right] \mathrm{d} s
$$

$P_{0}(t) \in \mathbb{R}^{n \times n}$ and $b_{0}(t) \in \mathbb{R}^{n}$ are the solutions of the following Riccati differential equation

$$
\left\{\begin{array}{l}
\dot{P}_{0}=-P_{0} A-A^{\mathrm{T}} P_{0}+P_{0} B R^{-1} B^{\mathrm{T}} P_{0}-Q, \quad P_{0}\left(t_{f}\right)=Q_{0} \\
\dot{b}_{0}=-\left[A-B R^{-1} B^{\mathrm{T}} P_{0}\right]^{\mathrm{T}} b_{0}-P_{0} \omega(t), \quad b_{0}\left(t_{f}\right)=0
\end{array}\right.
$$

Now, let us consider the influence from the unknown disturbance, the key idea is to completely eliminate the influence as much as possible based on a disturbance observer.

Theorem 3. Assume system (2) is a single input linear system, it is completely measurable and controllable. $v(t)$ is a matched unknown external disturbance satisfies $v(t)=B \gamma(t)$ and $\|\dot{v}(t)\| \leq d$, then we can design a delayed suboptimal trajectory tracking controller associates with performance criterion (3) as follows:

$$
u^{*}(t-\tau)=-R^{-1} B^{\mathrm{T}}\left[P_{0}(t) \bar{y}(t)+b_{0}(t)\right]-\mu B^{\mathrm{T}} \tilde{v}(t), \quad t \in\left[\tau, t_{f}\right]
$$

where $\bar{y}(t)$ is the predictor state of $y(t)$

$$
\bar{y}(t)=\mathrm{e}^{A \tau} y(t-\tau)+\int_{t-\tau}^{t} \mathrm{e}^{A(t-s)}\left[B u^{*}(s-\tau)+\omega(s)\right] \mathrm{d} s
$$

$\tilde{v}(t)$ is the estimated value of $v(t)$

$$
\left\{\begin{array}{l}
\tilde{v}(t)=\xi(t)+C \bar{y}(t) \\
\dot{\xi}(t)=-C \xi(t)-C\left(A \bar{y}(t)+B u^{*}(t-\tau)+\omega(t)+C \bar{y}(t)\right), \xi(0)=-C y(0)
\end{array}\right.
$$

$C$ is a positive definite symmetric matrix with large enough minimum eigenvalue, and $P_{0}(t) \in \mathbb{R}^{n \times n}$ and $b_{0}(t) \in \mathbb{R}^{n}$ are the solutions of the Riccati differential equation (34), $\mu=\frac{1}{B^{\mathrm{T} B}}$.

The designed controller, defined in (35) and shown in Figure 1, consists of two parts: $-R^{-1} B^{\mathrm{T}}\left[P_{0}(t) \bar{y}(t)+b_{0}(t)\right]$ is a controller to be responsible for the main control task, and the rest $\mu B^{\mathrm{T}} \tilde{v}$ is used for compensating the unknown disturbance $v(t)$. Each part performs its own function, and the two parts working together improve the robustness of the controller.

In summary, the designed controller is a combination of an optimal trajectory tracking controller and a disturbance observer, and can be obtained by the following steps.

Step 1. Transform the original system into an error system, where the given reference trajectory tracking target is converted into a known disturbance.

Step 2. Design an optimal controller for the error nominal system, where a linear quadratic performance criterion is introduced according to control task, and Riccati Eq.(34) is required to be solved.

Step 3. Design a disturbance observer, if unknown external disturbance is involved.

Step 4. Replace the current state in the designed controller with a predictor state, due to the delay effect. 


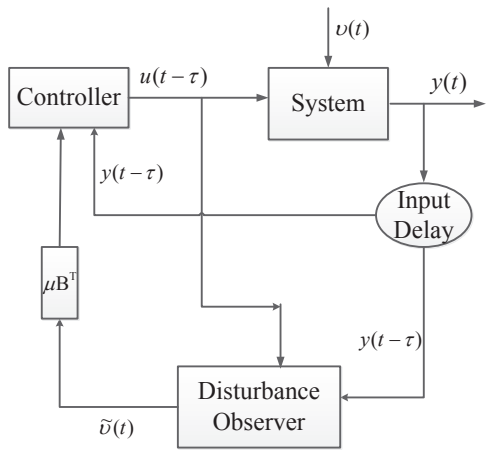

Figure 1: Structure of disturbance observer based control.

\section{Further discussion on the implementation of the proposed controller}

\subsection{Solution of the Riccati equation}

In designing the controller (35), the Riccati differential equation (34) should be solved firstly. This is usually not an easy task when the order of the system is high. For some special cases, the solution can be obtained analytically [9].

Actually, let us consider a linear differential equation

$$
\left[\begin{array}{c}
\dot{X}_{1}(t) \\
\dot{X}_{2}(t)
\end{array}\right]=H_{0}\left[\begin{array}{l}
X_{1}(t) \\
X_{2}(t)
\end{array}\right]
$$

subject to

$$
\left[\begin{array}{l}
X_{1}\left(t_{f}\right) \\
X_{2}\left(t_{f}\right)
\end{array}\right]=\left[\begin{array}{c}
I \\
Q_{0}
\end{array}\right]
$$

where the Hamiltonian matrix $H_{0}$ is defined by

$$
H_{0}=\left[\begin{array}{cc}
A & -B R^{-1} B^{\mathrm{T}} \\
-Q & -A^{\mathrm{T}}
\end{array}\right]
$$

The solution of the first equation in Eq.(34) is

$$
P_{0}(t)=X_{2}(t) X_{1}^{-1}(t)
$$

According to the virtues of the Hamiltonian matrix $H_{0}$, we know that if $\lambda_{0}$ is an eigenvalue of $H_{0}$, so is $-\lambda_{0}$. Moreover, $H_{0}$ has no pure imaginary eigenvalue due to the stabilizability of the original system. Therefore, there exists a transformation matrix $W$ such that

$$
W^{-1} H_{0} W=\left[\begin{array}{cc}
\Lambda_{s} & 0 \\
0 & \Lambda_{u}
\end{array}\right]
$$

where $\Lambda_{s} \in \mathbb{R}^{n \times n}, \Lambda_{u} \in \mathbb{R}^{n \times n}$ are real Jordan matrices with all eigenvalues possessing negative real and positive real part eigenvalues respectively. Let $W$ be rewritten in the form of block matrices as

$$
W=\left[\begin{array}{ll}
W_{11} & W_{12} \\
W_{21} & W_{22}
\end{array}\right]
$$


where the column vectors in the fist column block of $W$ are the eigenvectors of the eigenvalues of $H_{0}$ with negative real part, and the column vectors in the second column block of $W$ are the eigenvectors of the eigenvalues of $H_{0}$ with positive real part. Let

$$
\left[\begin{array}{l}
X_{1}(t) \\
X_{2}(t)
\end{array}\right]=W\left[\begin{array}{l}
\hat{X}_{1}(t) \\
\hat{X}_{2}(t)
\end{array}\right]
$$

then, Eq.(38) becomes

$$
\left[\begin{array}{c}
\hat{X}_{1}(t) \\
\hat{X}_{2}(t)
\end{array}\right]=\left[\begin{array}{cc}
\Lambda_{s} & 0 \\
0 & \Lambda_{u}
\end{array}\right]\left[\begin{array}{l}
\hat{X}_{1}(t) \\
\hat{X}_{2}(t)
\end{array}\right]
$$

Hence,

$$
\left\{\begin{array}{l}
\hat{X}_{1}(t)=\mathrm{e}^{-\Lambda_{s}\left(t_{f}-t\right)} \hat{X}_{1}\left(t_{f}\right) \\
\hat{X}_{2}(t)=\mathrm{e}^{-\Lambda_{u}\left(t_{f}-t\right)} \hat{X}_{2}\left(t_{f}\right)
\end{array}\right.
$$

From Eq.(39) and Eq.(40), we have

$$
\left\{\begin{array}{l}
I=W_{11} \hat{X}_{1}\left(t_{f}\right)+W_{12} \hat{X}_{2}\left(t_{f}\right) \\
M=W_{21} \hat{X}_{1}\left(t_{f}\right)+W_{22} \hat{X}_{2}\left(t_{f}\right)
\end{array}\right.
$$

Hence,

$$
\hat{X}_{2}\left(t_{f}\right)=E \hat{X}_{1}\left(t_{f}\right)
$$

where $E=-\left[W_{22}-M W_{12}\right]^{-1}\left[W_{21}-M W_{11}\right]$. According to Eq.(40), Eq.(41) and Eq.(42), we have

$$
\left\{\begin{array}{l}
X_{1}(t)=\left[W_{11}+W_{12} \mathrm{e}^{-\Lambda_{u}\left(t_{f}-t\right)} E \mathrm{e}^{\Lambda_{s}\left(t_{f}-t\right)}\right] \mathrm{e}^{-\Lambda_{s}\left(t_{f}-t\right)} \hat{X}_{1}\left(t_{f}\right) \\
X_{2}(t)=\left[W_{21}+W_{22} \mathrm{e}^{-\Lambda_{u}\left(t_{f}-t\right)} E \mathrm{e}^{\Lambda_{s}\left(t_{f}-t\right)}\right] \mathrm{e}^{-\Lambda_{s}\left(t_{f}-t\right)} \hat{X}_{1}\left(t_{f}\right)
\end{array}\right.
$$

Thus, the solution $P_{0}(t)$ can be rewritten as

$$
P_{0}(t)=T_{2}(t) T_{1}^{-1}(t)
$$

where

$$
\left\{\begin{array}{l}
T_{1}(t)=\left[W_{11}+W_{12} \mathrm{e}^{-\Lambda_{u}\left(t_{f}-t\right)} E \mathrm{e}^{\Lambda_{s}\left(t_{f}-t\right)}\right] \\
T_{2}(t)=\left[W_{21}+W_{22} \mathrm{e}^{-\Lambda_{u}\left(t_{f}-t\right)} E \mathrm{e}^{\Lambda_{s}\left(t_{f}-t\right)}\right]
\end{array}\right.
$$

The analytical formula (43) helps in finding the solution when the order of the system is not high. With $P_{0}(t)$ is in hand, $b_{0}(t)$ can be solved from the second (linear) equation in Eq.(34).

The design procedure can be greatly simplified for the special case: $t_{f} \rightarrow+\infty$, for which the quadratic performance criterion $J$ is in the form

$$
J=\frac{1}{2} \int_{0}^{+\infty}\left(y^{\mathrm{T}}(t) Q y(t)+u^{\mathrm{T}}(t) R u(t)\right) \mathrm{d} t
$$

and the first equation of Eq.(34) becomes an algebraic Riccati equation as follows

$$
-P_{0} A-A^{\mathrm{T}} P_{0}+P_{0} B R^{-1} B^{\mathrm{T}} P_{0}-Q=0
$$

In this case, the matrix $P_{0}$ is time-independent, and Eq.(34) becomes easier to solve.

\subsection{Numerical implementation}

From Eq.(35) and Eq.(36), the predictor feedback controller can be rewritten as

$$
\begin{aligned}
u^{*}(t-\tau) & =-R^{-1} B^{\mathrm{T}} P_{0}(t) \mathrm{e}^{A \tau} y(t-\tau)-R^{-1} B^{\mathrm{T}} b_{0}(t) \\
& -R^{-1} B^{\mathrm{T}} P_{0}(t) \int_{t-\tau}^{t} \mathrm{e}^{A(t-s)}\left[B u^{*}(s-\tau)+v(s)+\omega(s)\right] \mathrm{d} s-\mu B^{\mathrm{T}} \tilde{v}(t)
\end{aligned}
$$


It explicitly contains an integral term that is not practically feasible for online calculation. In the same way, this integral term is also should be dealt with when one make simulation experiment for system (2) under the controller (35) and the disturbance observer (37).

The numerical algorithm proposed in [29] can be used for implementing this controller. To this end, let

$$
U(t)=\int_{t-\tau}^{t} \mathrm{e}^{A(t-s)} B u^{*}(s-\tau) \mathrm{d} s
$$

and $\tau=N T-\rho$, where $T$ is the data sampling period, $N>0$ is a positive integer, and $0 \leq \rho<T$. The control forces exerted on system (2) can be considered as constants if the sampling period is sufficiently small,

$$
u^{*}(t)=u^{*}(k T), \quad k T \leq t<(k+1) T
$$

It follows that

$$
\begin{aligned}
U(t) & =\int_{-\tau}^{0} \mathrm{e}^{-A s} B u^{*}(s+t-\tau) \mathrm{d} s \\
& =\int_{-(N T-\rho)}^{-(N-1) T} \mathrm{e}^{-A s} B u^{*}(s+t-\tau) \mathrm{d} s+\int_{-(N-1) T}^{-(N-2) T} \mathrm{e}^{-A s} B u^{*}(s+t-\tau) \mathrm{d} s+\ldots+\int_{-T}^{0} \mathrm{e}^{-A s} B u^{*}(s+t-\tau) \mathrm{d} s \\
& =\mathrm{e}^{A(N T-\rho)} \int_{0}^{T-\rho} \mathrm{e}^{-A s_{1}} \mathrm{~d} s_{1} B u^{*}(t-2 N T+\rho)+\mathrm{e}^{A(N-1) T} \int_{0}^{T} \mathrm{e}^{-A s_{2}} \mathrm{~d} s_{2} B u^{*}(t-(2 N-1) T+\rho) \\
& +\ldots+\mathrm{e}^{A T} \int_{0}^{T} \mathrm{e}^{-A s_{N}} \mathrm{~d} s_{N} B u^{*}(t-T-N T+\rho)
\end{aligned}
$$

where the integrals can be calculated by using the regular fourth-order Runge-Kutta method. Moreover, by defining two functions

$$
\Psi_{1}(\delta)=\mathrm{e}^{A \delta}, \quad \Psi_{2}(\delta)=\int_{0}^{\delta} \mathrm{e}^{-A \eta} \mathrm{d} \eta B
$$

equation (46) can be rewritten as

$$
\begin{aligned}
U(t) & =\Psi_{1}(N T-\rho) \Psi_{2}(T-\rho) u^{*}(t-2 N T+\rho)+\Psi_{1}((N-1) T) \Psi_{2}(T) u^{*}(t-2 N T+T+\rho) \\
& +\ldots+\Psi_{1}(T) \Psi_{2}(T) u^{*}(t-T-N T+\rho)
\end{aligned}
$$

When $\rho=0$, Eq.(46) becomes

$$
U(t)=\Psi_{1}(N T) \Psi_{2}(T) u^{*}(t-2 N T)+\Psi_{1}((N-1) T) \Psi_{2}(T) u^{*}(t-2 N T+T)+\ldots+\Psi_{1}(T) \Psi_{2}(T) u^{*}(t-T-N T)
$$

The integral term $\Psi_{2}(\delta)$ can be determined from

$$
\Psi_{2}(\delta)=\int_{0}^{\delta} \mathrm{e}^{-A \eta} \mathrm{d} \eta B=\sum_{n=1}^{+\infty} \frac{(-A)^{n-1} \delta^{n}}{n !} B
$$

When $n$ is given, $\Psi_{2}(\delta)$ will converge to a constant matrix after limited steps of iterative calculation.

\section{Application to the control of Back-and-Forth motion of a cart-inverted pendulum}

Inverted pendulum mounted on a cart, shown in Figure 2, is an important mechanical model used for developing or checking control strategies. From the Lagrangian equations, its dynamic equation is given by

$$
\left\{\begin{array}{l}
(M+m) \ddot{s}-m l \ddot{\theta} \cos \theta+m l \dot{\theta}^{2} \sin \theta=F \\
\left(I+m l^{2}\right) \ddot{\theta}-m g l \sin \theta-m l \ddot{s} \cos \theta+b \dot{\theta}=0
\end{array}\right.
$$




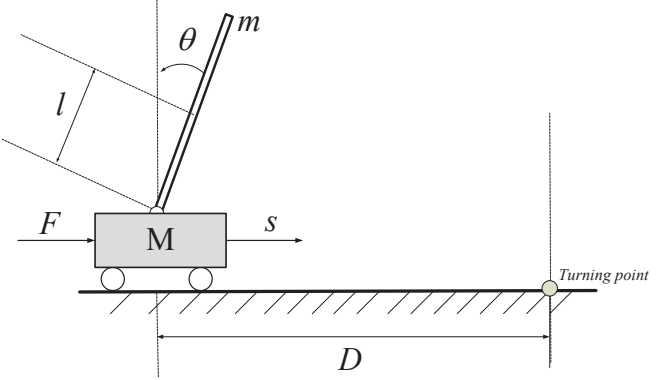

Figure 2: A simplified Back-and-Forth motion of the cart-inverted pendulum system.

where $M$ is the mass of the cart, $m$ is the mass of the pendulum, $s$ is the horizontal moving position of the cart, $l$ is the distance between joint and mass center of pendulum, $\theta$ is the vertical position angle, which can rotate in a plane around a point fixed on the cart, $g$ is the reduced gravity acceleration, $I$ is the moment of inertia of the pendulum, $b$ is the rotational damping ratio of the pendulum, $F$ is the horizontal force (control) performed on the cart, $\tau$ is the input delay reduced from measurement, computation and feedback, $D$ is the horizontal distance of the cart from the object. The inverted pendulum robot is to be designed to move forward to pass some prefixed point and return back to the starting point, keeping the inverted pendulum stabilized during the whole process.

With a large weight of the pitch angle error in the quadratic performance criterion, the control design can be made on the basis of the linearized model if the optimal trajectory tracking control strategy is applied. With a planned trajectory tracking target and minimized performance criterion, the optimal trajectory tracking controller forces the state variable to approach "imitatively" the tracking target via the Back-and-Forth motion, while the tilt angle of the pendulum is small enough and stabilized asymptotically to zero during the whole process. Assume that the rod of inverted pendulum is homogeneous, then the moment of inertia is $I=\frac{1}{3} m l^{2}$, and the linearized equation of the inverted pendulum with an input delay is given by

$$
\left\{\begin{array}{l}
(M+m) \ddot{s}-m l \ddot{\theta}=F(t-\tau) \\
\frac{4}{3} m l^{2} \ddot{\theta}-m g l \theta-m l \ddot{s}+b \dot{\theta}=0
\end{array}\right.
$$

With $p=\frac{M}{m}, N=\frac{4}{3} p+\frac{1}{3}$, this equation is simplified to

$$
\left\{\begin{array}{l}
\ddot{\theta}=\frac{p+1}{N} g \theta-\frac{b(p+1)}{N m l} \dot{\theta}+\frac{1}{N m} F(t-\tau) \\
\ddot{s}=\frac{g}{N} \theta-\frac{b}{N m l} \dot{\theta}+\frac{4}{3 N m} F(t-\tau)
\end{array}\right.
$$

Let $\mathbf{x}=\left[x_{1}, x_{2}, x_{3}, x_{4}\right]^{\mathrm{T}}=[\theta, \dot{\theta}, s, \dot{s}]^{\mathrm{T}}, u(t-\tau)=F(t-\tau)$, and

$$
A=\left[\begin{array}{cccc}
0 & 1 & 0 & 0 \\
\frac{p+1}{N} g & -\frac{b(p+1)}{N m l} & 0 & 0 \\
0 & 0 & 0 & 1 \\
\frac{g}{N} & -\frac{b}{N m l} & 0 & 0
\end{array}\right], \quad B=\left[\begin{array}{c}
0 \\
\frac{1}{N m} \\
0 \\
\frac{4}{3 N m}
\end{array}\right]
$$

then, the simplified inverted pendulum with delayed input can be described in state equation

$$
\dot{\mathbf{x}}(t)=A \mathbf{x}(t)+B u(t-\tau), \quad \mathbf{x}(0)=[0,0,0,0]^{\mathrm{T}}
$$


For demonstration, let

$$
\begin{aligned}
& Q=\operatorname{diag}(100,0,100,0), \quad R=1, \quad M=11 \mathrm{~kg}, \quad m=1 \mathrm{~kg}, \quad l=1 \mathrm{~m}, \quad g=10 \mathrm{~m} / \mathrm{s}^{2}, \\
& \tau=0.05 s, \quad D=3.2 \mathrm{~m}, \quad b=0.1, \quad t_{f}=+\infty, \quad \theta(0)=0, \quad \dot{\theta}(0)=0, \quad s(0)=0, \quad \dot{s}(0)=0 .
\end{aligned}
$$

Then the matrices $A$ and $B$ in Eq.(49) become

$$
A=\left[\begin{array}{cccc}
0 & 1 & 0 & 0 \\
8 & -0.08 & 0 & 0 \\
0 & 0 & 0 & 1 \\
\frac{2}{3} & -\frac{1}{150} & 0 & 0
\end{array}\right], \quad B=\left[\begin{array}{c}
0 \\
\frac{1}{15} \\
0 \\
\frac{4}{45}
\end{array}\right]
$$

Under such a parameter combination, the trajectory tracking target can be chosen carefully to be $[\bar{\theta}(t), \bar{s}(t)]^{\mathrm{T}}=[0,(a t-$ $\left.\left.t^{2}\right) \mathrm{e}^{-\alpha t}\right]^{\mathrm{T}}$ with $a=36, \alpha=1$. Therefore, the trajectory tracking target vector $\overline{\mathbf{x}}$ and the vector $\omega(t)$ are as follows:

$$
\begin{aligned}
& \overline{\mathbf{x}}=\left[\bar{x}_{1}, \bar{x}_{2}, \bar{x}_{3}, \bar{x}_{4}\right]^{\mathrm{T}}=[\bar{\theta}, \dot{\bar{\theta}}, \bar{s}, \dot{\bar{s}}]^{\mathrm{T}}=\left[0,0,\left(36 t-t^{2}\right) \mathrm{e}^{-t},\left(36-38 t+t^{2}\right) \mathrm{e}^{-t}\right]^{\mathrm{T}} \\
& \omega(t)=A \overline{\mathbf{x}}-\dot{\overline{\mathbf{x}}}=\left[0,0,0,\left(74-40 t+t^{2}\right) \mathrm{e}^{-t}\right]^{\mathrm{T}}
\end{aligned}
$$

The control design is based on the linearized model of the inverted pendulum with strong nonlinearity, and the modelling error caused by linearization contains quadratic terms, cubic terms, and higher-order nonlinear terms, with respect to $\theta$. If $\theta$ is not small, then the high order terms have important effect on the modelling error and controller design. Large $\theta$ results in unsuccessful. If $\theta$ is small, then the modelling error is dominated by the quadratic nonlinear terms. The pecularity of the proposed method is the introduction of a quadratic performance with large weight of $\theta$ 's error to make $\theta$ small enough in the whole motion process. Thus, it is reasonable to assume that the uncertainties from the the tilt angle position and tilt angle velocity are in the following matched form

$$
v(t)=B\left(c_{1} \theta^{2}+c_{2} \dot{\theta}^{2}+c_{3} \theta \dot{\theta}\right)
$$

Let $\mathbf{y}(t)=\mathbf{x}(t)-\overline{\mathbf{x}}(t)=\left[y_{1}, y_{2}, y_{3}, y_{4}\right]^{\mathrm{T}}$, then $\mathbf{y}(t)$ is governed by

$$
\left\{\begin{array}{l}
\dot{\mathbf{y}}(t)=A \mathbf{y}(t)+B u(t-\tau)+v(t)+\omega(t) \\
\mathbf{y}(0)=\mathbf{x}(0)-\overline{\mathbf{x}}(0)=[0,0,0,-36]^{\mathrm{T}}
\end{array}\right.
$$

For the case of $t_{f} \rightarrow+\infty$, the quadratic performance criterion $J$ is in the following form

$$
J=\frac{1}{2} \int_{0}^{+\infty}\left(\mathbf{y}^{\mathrm{T}}(t) Q \mathbf{y}(t)+u^{\mathrm{T}}(t) R u(t)\right) \mathrm{d} t
$$

The first matrix Riccati differential equations of Eq.(34) become algebraic Riccati equation

$$
-P_{0} A-A^{\mathrm{T}} P_{0}+P_{0} B R^{-1} B^{\mathrm{T}} P_{0}-Q=0
$$

Then, the MATLAB command lqr returns the Riccati matrix $P_{0}$ and $b_{0}(t)$ for the linear system (50) as follows:

$$
P_{0}=\left[\begin{array}{cccc}
25761 & 9154 & -1372 & -2610 \\
9154 & 3255 & -494.4 & -940.6 \\
-1372 & -494.4 & 227.3 & 258.3 \\
-2610 & -940.6 & 258.3 & 449.8
\end{array}\right], \quad b_{0}(t)=\left[\begin{array}{c}
\left(-1509 t^{2}+58844 t-83287\right) \mathrm{e}^{-t} \\
\left(-544 t^{2}+21207 t-30016\right) \mathrm{e}^{-t} \\
\left(118.5 t^{2}-4656 t+7196\right) \mathrm{e}^{-t} \\
\left(250.8 t^{2}-9788 t+14054\right) \mathrm{e}^{-t}
\end{array}\right]
$$

The designed controller is given by

$$
u^{*}(t-\tau)=-R^{-1} B^{\mathrm{T}}\left[P_{0}(t) \overline{\mathbf{y}}(t)+b_{0}(t)\right]-\mu B^{\mathrm{T}} \tilde{v}(t)
$$




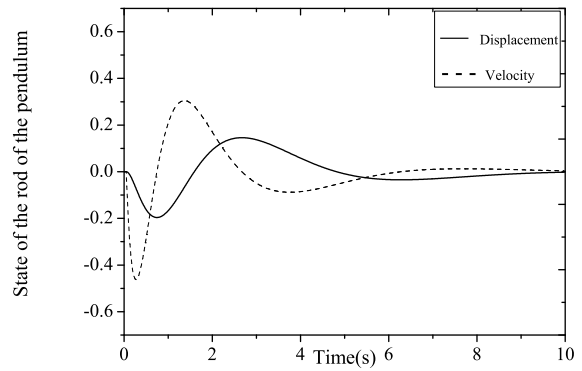

(a) The time history of the state of the rod of pendulum

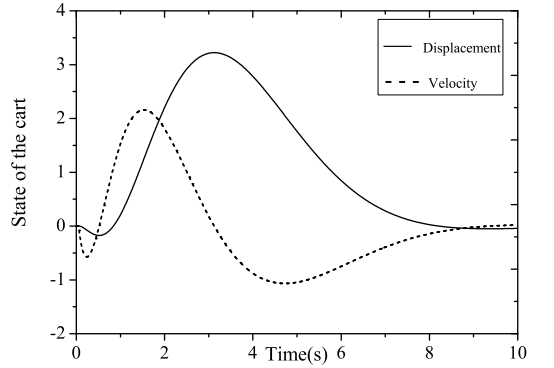

(b) The time histories of the state of the cart

Figure 3: The motion state of the inverted cart-pendulum under the optimal trajectory tracking controller with disturbance observer

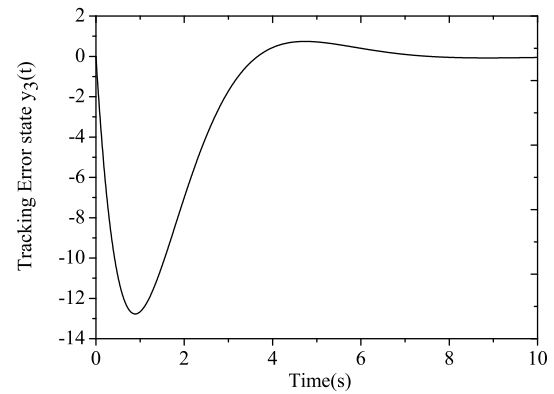

(a) Displacement tracking error of the cart

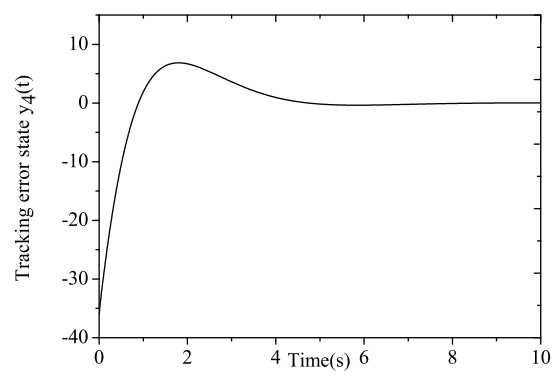

(b) Velocity tracking error of the cart

Figure 4: The time histories of the tracking error of the cart.

where $\overline{\mathbf{y}}(t)$ is the predictor state of $\mathbf{y}(t)$

$$
\overline{\mathbf{y}}=\mathrm{e}^{A \tau} \mathbf{y}(t-\tau)+\int_{t-\tau}^{t} \mathrm{e}^{A(t-s)}\left[B u^{*}(s-\tau)+\omega(s)\right] d s
$$

and $\tilde{v}(t)$ is the estimated value of $v(t)$ given by

$$
\left\{\begin{array}{l}
\tilde{v}=\xi+C \bar{y} \\
\dot{\xi}=-C \xi-C\left(A \bar{y}+B u^{*}(t-\tau)+\omega(t)+C \bar{y}\right), \xi(0)=-C y(0)
\end{array}\right.
$$

In simulation, the invertible matrix $C$ is taken as $C=\operatorname{diag}(100,100,100,100)$, and $\mu=\left(B^{\mathrm{T}} B\right)^{-1}=81$. Then, all the quantities required in the designed controller $u^{*}(t-\tau)$ are available in hand. In addition, the coefficients $c_{1}, c_{2}, c_{3}$ in the unknown disturbance $v(t)$ are set to be $c_{1}=c_{2}=20, c_{3}=10$. As seen in Figure 3(a), the pitch angle of the inverted pendulum is small and stabilized during the Back-and-Forth motion of the cart, while Figure 3(b) shows that the cart moves forward $3.2 \mathrm{~m}$ to the terminal point and returns back to the starting point within 10 seconds, and that this motion process consists of four stages: reversed accelerating $\longrightarrow$ forward accelerating $\longrightarrow$ reversed accelerating $\longrightarrow$ forward accelerating. This is in full agreement with the actual situation. Figure 4 shows the tracking error of the cart, which converges to zero quickly. 


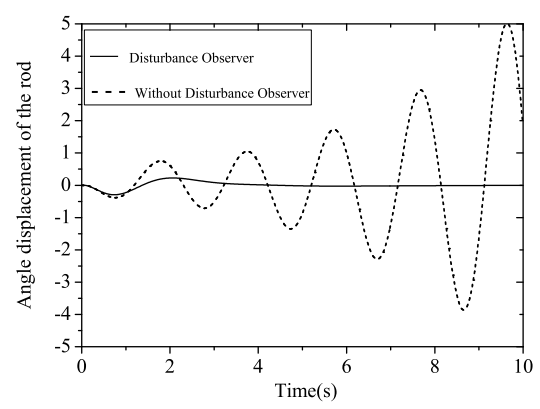

Figure 5: The time history of the pitch angle of the rod of pendulum under the optimal trajectory tracking controller with/without disturbance observer

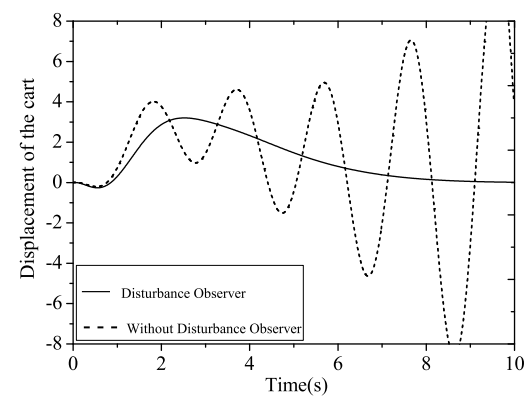

(a)Displacement of the cart

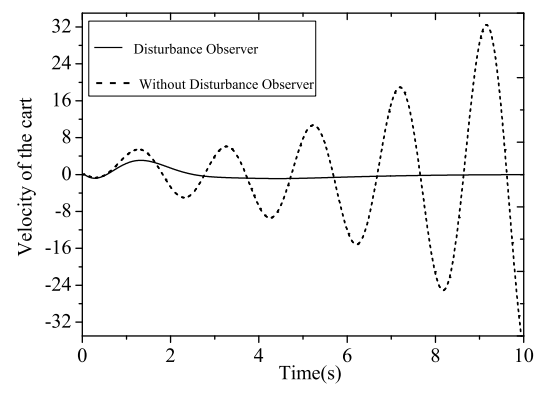

(b) Velocity of the cart

Figure 6: The time histories of the state of the cart under the optimal trajectory tracking controller with/without disturbance observer

The Back-and-Forth motion of the inverted pendulum system might be designed by using regular tracking control theory. However, the regular tracking controller cannot guarantee the pitch angle of the inverted pendulum keeping small when the cart is moving forward, thus the inverted pendulum can be easily collapsed or the control problem can not be solved by using linear theory.

The weight matrix $Q$ plays an important role in the implementation of the Back-and-Forth motion. It restrains the motion of the rod of pendulum, and makes the cart to track the reference displacement as far as possible. In the above numerical example, the weight of the pitch angle error is taken as the same as the weight of the cart displacement error. If the weight of the pitch angle error is increased, the maximum value of the pitch angle of the rod of pendulum will be decreased. Hence, the increase of the weight of the pitch angle error leads to better performance of the whole motion process, but yields a shorter maximal displacement of the cart. In order to arrive at turning point, the parameter value $a$ should be increased. If one increases the weight of the cart displacement error only, the motion range of the pitch angle will be increased, and this may lead to system collapse under the optimal trajectory tracking $\operatorname{control} u^{*}(t-\tau)$. Thus, the matrix $Q$ must be carefully chosen.

Figure 5-6 show that unknown disturbance has great influence to the control effect of the optimal trajectory tracking control. Without a disturbance observer, the optimal trajectory tracking controller makes the controlled system not convergent. With the disturbance observer, however, the designed controller not only improves greatly the robustness of optimal trajectory tracking control, but also retains the control effect of the optimal control. 


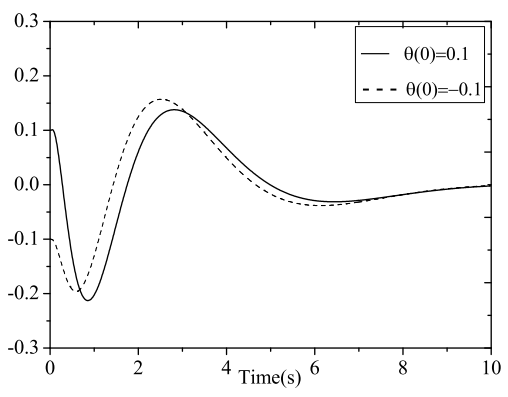

(a) $\theta(0)=0.1, \dot{\theta}(0)=0$ and $\theta(0)=-0.1, \dot{\theta}(0)=0$

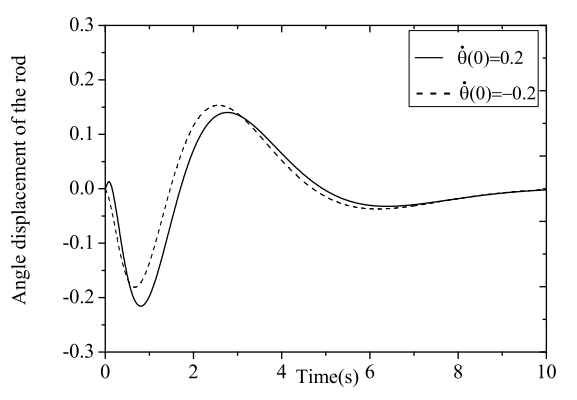

(b) $\theta(0)=0, \dot{\theta}(0)=0.2$ and $\theta(0)=0, \dot{\theta}(0)=-0.2$

Figure 7: The comparisons of the time histories of the pitch angle of the pendulum under different tilt angle initial conditions

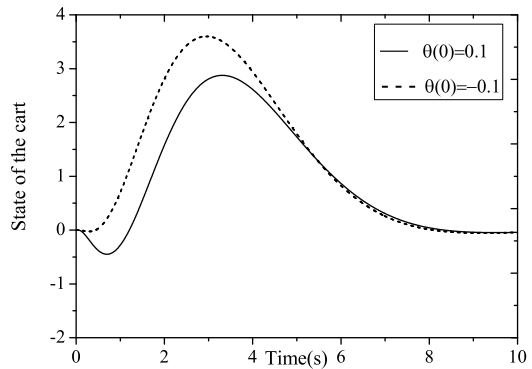

(a) $\theta(0)=0.1, \dot{\theta}(0)=0$ and $\theta(0)=-0.1, \dot{\theta}(0)=0$

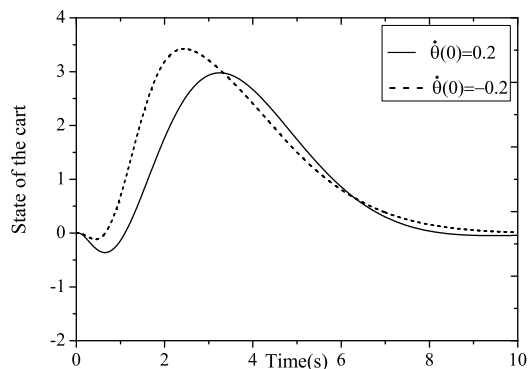

(b) $\theta(0)=0, \dot{\theta}(0)=0.2$ and $\theta(0)=0, \dot{\theta}(0)=-0.2$

Figure 8: The comparisons of the time histories of the displacement of the cart under different tilt angle initial conditions

The pendulum is required to keep stable during the cart's motion. Thus, it is expected to have a small initial pitch angle and a small initial angular velocity. In order to make the cart moving forward and keep the pitch angle small enough simultaneously, the pendulum is required to be a negative value. Thus, the cart must have an accelerating to the opposite direction at the beginning of the movement, as shown in Figure 3(a). Therefore, the initial conditions with a negative pitch angle of the pendulum can improve the control effect, as validated in Figures 7-8.

In addition, the initial velocity of the cart has a major influence on the control effect, positive effect or negative effect as shown in Figure 9(a) with positive initial velocity or with negative initial velocity. While in Figure 9(b) with a positive initial velocity, the maximal displacement of the cart is larger than the prefixed distance $D=3.2$ defined in Figure 2, and it can be adjusted to a smaller one by decreasing the parameter value $a$ in the trajectory tracking target. However, with negative initial velocity, the cart failed to reach the turning point, an increasing of the parameter value $a$ is necessary, this in turn leads to a larger pitch angle of the pendulum. Nevertherless, due to the nature of inverted pendulum on a cart, self-balancing should be firstly achieved. Thus, the initial values can be assumed small in all numerical simulation. 


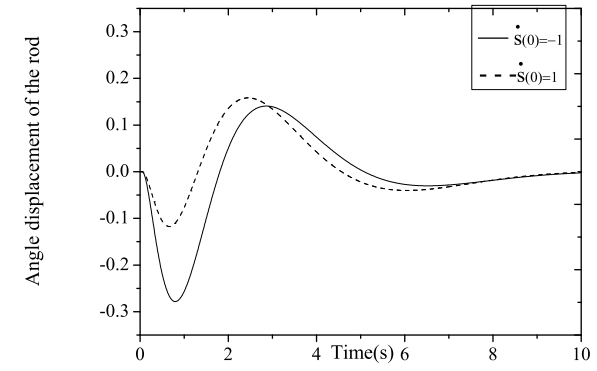

(a) $\dot{s}(0)=-1$ and $\dot{s}(0)=1$

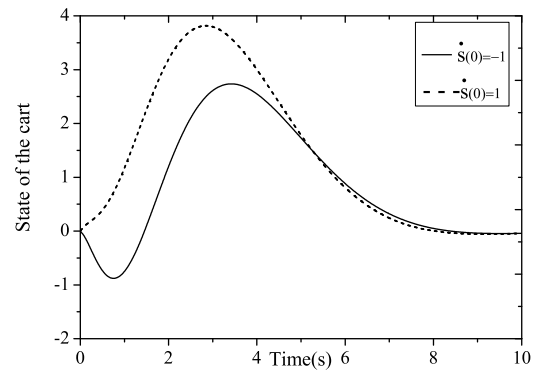

(b) $\dot{s}(0)=-1$ and $\dot{s}(0)=1$

Figure 9: The comparisons of the control effect of the proposed controller under different initial velocity of the cart

\section{Conclusions}

The contribution of this paper is two folds. One is the introduction of a new integral state transformation that is used to convert the delayed system to a delay-free one, which results in the simple relationship between the original state variable and the new state variable. Thus, the delayed optimal trajectory tracking controller is designed by using a predictor-based feedback in terms of the original state variables, unlike in the applications of the conventional transformation widely used in the literature. The other is the introduction of the disturbance observer in the closed-loop for compensating the inevitable unknown disturbance timely. As shown in the control of Back-and-Forth motion of an inverted pendulum on a cart, the designed controller not only improves greatly the robustness of optimal trajectory tracking control, but also retains the control effect of the optimal control. In a sense, the proposed design method gives a solution to overcome the fatal flaw of the optimal control theory: bad robustness.

Acknowledgments. The authors thank the financial support of NSF of China under Grant 11372354, Funding of Jiangsu Innovation Program for Graduate Education (CXLX13-129), and thank the anonymous reviewers for their valuable comments that improve the presentation of this paper.

\section{References}

[1] M. Chadli, S. Aouaouda, H.R. Karimi, P. Shi, Robust fault tolerant tracking controller design for a VTOL aircraft, J. Frankl. Inst. $350(9)(2013) 2627-2645$.

[2] G. Gao, J.Z. Wang, Reference command tracking control for an air-breathing hypersonic vehicle with parametric uncertainties, J. Frankl. Inst. $350(5)(2013) 1155-1188$.

[3] M.Y. Ou, H.B. Du, S.H. Li, Finite-time tracking control of multiple nonholonomic mobile robots, J. Frankl. Inst. 349(9)(2012)2834-2860.

[4] Q.L. Hu, L. Xu, A.H. Zhang, Adaptive backstepping trajectory tracking control of robot manipulator, J. Frankl. Inst. 349(3)(2012)1087-1105.

[5] H. Ghorbel, A. Hajjaji, M. Souissi, M. Chaabane, Robust tracking control for takagi-sugeno fuzzy systems with unmeasurable premise variables: application to tank system, ASME J. Dyn. Syst. Meas. Contr. 136(2014)041011.

[6] B.S. Park, Neural network-based tracking control of underactuated autonomous underwater vehicles with model uncertainties, ASME J. Dyn. Syst. Meas. Contr. 137(2015)021004.

[7] M. Santhakumar, J. Kim, Robust adaptive tracking control of autonomous underwater vehicle-manipulator systems, ASME J. Dyn. Syst. Meas. Contr. 136(2014)054502.

[8] S.P. Sethi£G.L. Thompson, Optimal Control Theory: Applications to Management Science and Economics, Springer, 2006.

[9] B.O. Anderson, J.B. Moore, Optimal Control: Linear Quadratic Methods, Prentice Hall Inc., Englewood cliffs, New Jersey, 1989

[10] S.K. Biswas, S.J. Chen, A. Satyanarayana, Optimal temperature tracking control for accelerated cooling processes in hot rolling of steel, Dynamics and Control 7(1997)327-340.

[11] T. Cimen, S.P. Banks, Nonlinear optimal tracking control with application to super-tankers for autopil design, Automatica 40(11)(2004)18451863. 
[12] K.D. Do, J. Pan, State and output-feedback robust path-follwing controllers for underactuated ships using street-frenet frame, Ocean Engineering 31(2004)587-613.

[13] M.S. Lee, Y.H. Kim, Automotive radar tracking of multi-target for vehicle CW/CA systems, Mechatronics 14(2004)143-151.

[14] E. Devaud, S. Font, Some control strategies for a hign-angle-of-attack missile autopilot, Control Engineering Practice 8(2000)885-992.

[15] R. Sharma, A. Tewari, Optimal nonlinear tracking of spacecraft attitude maneuvers, IEEE Trans. Control System Tech. 12(2004)677-682.

[16] T.S. No, B.M. Min, R.H. Stone, K.C. Wong, Control and simulation of arbitrary flight trajectory tracking, Control Engineering Practice 13(2005)601-612

[17] M.T. Grabbe, D.M. Dawson, Application of optimal control theory to the trajectory tracking of rigid robot manipulators, Optimal Control Appl. Method. 15(1994)237-249.

[18] P. Tomei, Tracking control of flexible joint robots with uncertain parameters and disturbance, IEEE Trans. Autom. Control. 39(1994)10671072.

[19] B. Liu, H.Y. Hu, Group delay induced instability and its suppression for a controlled-double pendulum, J. Vib. Engi. 5(2009)128-135.

[20] J.P. Richard, Time-delay systems: an overview of some recent advances and open problems, Automatica 39(2003)1667-1694.

[21] J.D. Chen, LMI-based robust $H_{\infty}$ control of uncertain neutral systems with state and input delays. J. Optim. Theory Appl. 126(3)(2005)553570

[22] M.S. Mahmoud, M. Zribi, $H_{\infty}$ controllers for time-delay systems using linear matrix inequalities. J. Optim. Theory Appl. 100(1)(1999)89-122

[23] W.H. Kwon, A.E. Pearson, Feedback stabilization of linear systems with delayed control, IEEE Trans. Autom. Control 25(1980)266-269.

[24] Z. Arstein, Linear systems with delayed control: a reduction, IEEE Trans. Autom. Control 27(1982)869-879.

[25] J.D. Han, Y.Q. He, X.G. Zhao, Mobile Robotics Systems: Modelling, Estimation and Control, Science Press, Beijing, 2011.

[26] S. Sastry, Nonlinear Systems: Analysis, Stability and Control, Springer-Verlag, New York, 1999.

[27] W.H. Chen, Disturbance observer based control for nonlinear systems, IEEE Trans. Mechatronics 9(4)(2004)706-710

[28] K.D. Do, G. Seet, Motion control of a two-wheeled mobile vehicle with an inverted pendulum, J. Intell. Robotic Syst. 60(3-4)(2010)577-605

[29] G.P. Cai, J.Z. Huang, S.X. Yang, An optimal control method for linear systems with time delay, Comput. and Struct. 81(2003)1539-1546.

[30] L. Behera, I. Kar, Intelligent Systems and control principles and applications, Oxford University Press, Inc., 2010.

[31] G.L. Baker, J.A. Blackburn, The Pendulum. Oxford University Press, 2005. 